\title{
PERFECT SEMIGROUPS
}

\author{
by JOHN FOUNTAIN
}

(Received 22nd October 1974)

\section{Introduction}

A semigroup $S$ with identity is (left) perfect if every unitary left $S$-system has a projective cover. This is the semigroup analogue of the definition of left perfect rings introduced in (1). The investigation of perfect semigroups was initiated by Isbell (4), who proved that a semigroup is perfect if and only if it satisfies two conditions referred to as conditions $A$ and $D$.

Left perfect rings have several equivalent characterisations, one of which is that the rings satisfy the descending chain condition for principal right ideals. This is not enough for perfection of a semigroup as is shown by an example in (4). However, Isbell conjectures that in the presence of condition $A$, a semigroup satisfies condition $D$ if and only if it satisfies $M_{R}$, the minimum condition for principal right ideals. One of the objects of this note is to show that this conjecture is correct. In fact, more is true. Björk (2) has shown that if a module satisfies the descending chain condition for cyclic submodules, then it also satisfies the descending chain condition for finitely generated submodules. The corresponding semigroup result is true with a similar but easier proof. Thus perfect semigroups satisfy the minimum condition for finitely generated right ideals.

Another characterisation of left perfect rings is that every direct limit of projective modules is projective. Isbell has shown that for semigroups the corresponding condition is sufficient but has left open the question of necessity. Following Strenström (7) we shall call an $S$-system which is a direct limit of projective $S$-systems, a weakly flat $S$-system. Such $S$-systems have also been considered by Kil'p in (5), where they are called strongly flat. Strenstöm gives several characterisations of weakly flat $S$-systems, one of which provides us with the main tool for our investigations. Using this, we show that for a perfect semigroup $S$, every weakly flat $S$-system is projective, thus answering the question of Isbell.

\section{Preliminaries and statement of theorem}

All semigroups considered have an identity. For a semigroup $S$, a left $S$-system is a set $A$ together with a function $S \times A \rightarrow A:(s, x) \rightarrow s x$ satisfying $s(t x)=(s t) x$ and $1 x=x(x \in A, s, t \in S)$. Right $S$-systems are defined in a similar way. We shall refer to left $S$-systems simply as $S$-systems. A subsystem of an $S$-system $A$ is a subset $B$ of $A$ which satisfies $S B \subseteq B$. Let $A$ and $B$ be 
two $S$-systems. A function $f: A \rightarrow B$ is called an $S$-homomorphism if $f(s x)=s f(x)$ for all $s \in S, x \in A$.

A free $S$-system is simply a disjoint union of copies of $S$ and a projective $S$-system is a retract of a free $S$-system. A projective cover of an $S$-system $A$ is a surjective $S$-homomorphism $f: P \rightarrow A$, where $P$ is a projective $S$-system and the restriction of $f$ to any proper subsystem of $P$ is not surjective.

A subset $X$ of an $S$-system $A$ is a set of generators for $A$ if $A=S X$ and is a free set of generators if also $S x \cap S x^{\prime}=\varnothing$ for $x, x^{\prime} \in X, x \neq x^{\prime}$ and $S x \approx S$ for $x \in X$.

An $S$-system $A$ is decomposable if there are non-empty subsystems $B, C$ of $A$ such that $A=B \cup C$ and $B \cap C=\varnothing$. Otherwise, $A$ is called indecomposable. Clearly a cyclic $S$-system is indecomposable. From (6), we have the following two results.

Result 1. Every S-system is uniquely decomposable into a disjoint union of indecomposable subsystems.

Result 2. A disjoint union of $S$-systems $P_{i}$ is projective if and only if each $P_{i}$ is projective. An $S$-system $P$ is an indecomposable projective if and only if $P \approx S e$ for some idempotent $e$ in $S$.

The tensor product $A \otimes B$ of a right $S$-system $A$ and an $S$-system $B$ is the set $(A \times B) / \sim$, where $\sim$ is the equivalence relation generated by $(a s, b) \sim(a, s b)$ for $a \in A, b \in B, s \in S$. The $S$-system $B$ is weakly flat if the functor $\otimes B$ preserves equalisers and pullbacks. As noted in (5) and (7), projective $S$-systems are weakly flat. From (7) we also have the following two results.

Result 3. A direct limit of weakly flat $S$-systems is weakly flat.

Result 4. The following properties of an S-system $A$ are equivalent:

(i) $A$ is weakly flat.

(ii) If $s x=$ ty where $x, y \in A$ and $s, t \in S$, then there is an element $z$ in $A$ and elements $s^{\prime}, t^{\prime}$ in $S$ such that $x=s^{\prime} z, y=t^{\prime} z$ and $s s^{\prime}=t t^{\prime} ;$ moreover, if $x=y$, we may take $s^{\prime}=t^{\prime}$.

(iii) $A$ is a direct limit of finitely generated free systems.

An easy consequence of Results 1 and 4 is that every weakly flat $S$-system is a disjoint union of indecomposable weakly flat $S$-systems.

From (3, chapter 11) we have that a cyclic $S$-system is isomorphic to $S / \rho$ where $\rho$ is a left congruence on $S$. We call $\rho$ weakly flat if $S / \rho$ is a weakly flat $S$-system. As a corollary of Result 4, we have

Corollary. For a left congruence $\rho$ on $S$, the following are equivalent:

(i) $\rho$ is weakly flat.

(ii) If sot where $s, t \in S$, then there are elements $u, v \in S$ such that $s u=t u$ and $1 \rho u v$. 
Proof. The implication (i) $\Rightarrow$ (ii) is immediate from (ii) of Result 4.

For (ii) $\Rightarrow($ i), let $a \rho, b \rho \in S / \rho$ and suppose that $s(a \rho)=t(b \rho)$. Then $s a \rho t b$ so that $s a u=t b u$ and $1 \rho u v$ for $u, v \in S$, that is, $s s^{\prime}=t t^{\prime}$ and

$$
a \rho=s^{\prime}(v \rho), b \rho=t^{\prime}(v \rho) \text {, }
$$

where $s^{\prime}=a u, t^{\prime}=b u$. Thus (ii) of Result 4 is satisfied.

From (4) we have

Result 5. For a subset $B$ of $S$, we have $B=1 \rho$ for some left congruence $\rho$ on $S$ if and only if $B$ is a left unitary subsemigroup of $S$.

Condition $A$ in (4) states that every locally cyclic $S$-system is cyclic or equivalently every $S$-system satisfies the ascending chain condition for cyclic subsystems.

Condition $D$ in (4) states that every left unitary subsemigroup of $S$ has a minimal left ideal generated by an idempotent.

The main theorem can now be stated as follows.

Theorem. For a semigroup $S$, the following are equivalent:

(i) $S$ is perfect.

(ii) $S$ satisfies conditions $A$ and $D$.

(iii) $S$ satisfies conditions $A$ and $M_{R}$.

(iv) $S$ satisfies condition $A$ and the descending chain condition for finitely generated right ideals.

(v) Every weakly flat S-system is projective.

\section{Proof of the main theorem}

The equivalence (i) $\Leftrightarrow$ (ii) is due to Isbell and in view of Result 4 so is the implication (v) $\Rightarrow$ (ii). The implication (iv) $\Rightarrow$ (iii) is obvious. We shall prove the implications (v) $\Rightarrow$ (iii), (ii) $\Rightarrow$ (v), (iii) $\Rightarrow$ (v) and (iii) $\Rightarrow$ (iv).

Lemma 1. If every weakly flat $S$-system is projective, then $S$ satisfies $M_{R}$.

Proof. If $a_{1} S \supseteq b_{2} S \supseteq b_{3} S \supseteq \ldots$ is a decreasing sequence of principal right ideals of $S$, then $b_{2}=a_{1} a_{2}, b_{3}=a_{1} a_{2} a_{3}, \ldots$ for some elements $a_{2}, a_{3}, \ldots$ in $S$. Let $F$ be the free $S$-system freely generated by $x_{1}, x_{2}, \ldots, x_{n}, \ldots$ and let $\rho$ be the congruence on $F$ generated by the relation

$$
\sigma=\left\{\left(x_{i}, a_{i} x_{i+1}\right): i=1,2, \ldots\right\} .
$$

Then $\rho$ is the transitive closure of the relation $\theta$ where

$$
\theta=\left\{\left(s x_{i}, s a_{i} x_{i+1}\right),\left(s a_{i} x_{i+1}, s x_{i}\right),\left(s x_{i}, s x_{i}\right): s \in S, \quad i=1,2, \ldots\right\} .
$$

We show first that for elements $s, t$ of $S, s x_{n} \rho t x_{n}$ if and only if there is a nonnegative integer $m$ such that $s a_{n} \ldots a_{n+m}=t a_{n} \ldots a_{n+m}$. If we have

$$
s a_{n} \ldots a_{n+m}=t a_{n} \ldots a_{n+m},
$$


then clearly $s x_{n} \rho t x_{n}$. Suppose that $s x_{n} \rho t x_{n}$. Then $s^{\prime} x_{h} \rho t^{\prime} x_{k}$ where if $s \notin S a_{n-1}$, then $h=n, s^{\prime}=s$ and if $s \in S a_{n-1}$, then $h$ is the smallest positive integer such that $s \in S a_{h} \ldots a_{n-1}$ and $s^{\prime} \in S$ is such that $s=s^{\prime} a_{h} \ldots a_{n-1}$ and $k, t^{\prime}$ are obtained similarly from $t$. Thus there are elements $y_{1}, \ldots, y_{p}$ of $F$ such that

$$
s^{\prime} x_{h}=y_{1}, t^{\prime} x_{k}=y_{p} \text { and } y_{r} \theta y_{r+1}(r=1, \ldots, p-1) \text {. }
$$

From the definitions of $\theta, s^{\prime}, t^{\prime}$ we have that for some $r$,

$$
y_{r}=s^{\prime} a_{h} \ldots a_{i} x_{i+1}, y_{r+1}=t^{\prime} a_{k} \ldots a_{j} x_{j+1}
$$

and one of $i=j+1, i=j$ or $i+1=j$ holds. Whichever one holds we certainly obtain $s^{\prime} a_{h} \ldots a_{i+1}=t^{\prime} a_{k} \ldots a_{i+1}$ and the desired conclusion follows.

Now for each positive integer $n$ let $\rho_{n}$ be the congruence on $F$ generated by the relation

$$
\sigma_{n}=\left\{\left(x_{1}, a_{1} x_{2}\right), \ldots,\left(x_{n}, a_{n} x_{n+1}\right)\right\} .
$$

If $i, j>n$ and $s x_{i} \rho_{n} t x_{j}$, then $i=j$ and $s=t$. If $i \leqq n$, then $x_{i} \rho_{n} a_{i} \ldots a_{n} x_{n+1}$. Since $\left\{x_{i} \rho_{n}: i=1,2, \ldots\right\}$ is clearly a set of generators for $F / \rho_{n}$, it follows that $\left\{x_{i} \rho_{n}: i=n+1, n+2, \ldots\right\}$ is a free set of generators for $F / \rho_{n}$. Clearly $\rho_{i} \subseteq \rho_{i+1} \subseteq \rho$ for all $i=1,2, \ldots$ Thus for $m<n, \alpha_{m, n}: F / \rho_{m} \rightarrow F / \rho_{n}$ which takes $x \rho_{m}$ to $x \rho_{n}$ is a surjective $S$-homomorphism, as is $\alpha_{m}: F / \rho_{m} \approx F / \rho$ which takes $x \rho_{m}$ to $x \rho$, and $\alpha_{n} \alpha_{m, n}=\alpha_{m}$. Furthermore, if $x, y \in F$, say $x=s x_{k}$, $y=t x_{j}, j \leqq k$ and $x \rho_{m}, y \rho_{n}$ have the same image in $F / \rho$, then $x \rho y$ so that $s x_{k} \rho t a_{j} \ldots a_{k-1} x_{k}$ and so $s a_{k} \ldots a_{k+q}=t a_{j} \ldots a_{k+q}$ for some $q$ giving $x \rho_{k+q} y$. Hence for some $r \geqq m, n$ we have $x \rho_{r}=y \rho_{r}$ and it follows that $F / \rho$ is the direct limit of the free $S$-systems $F / \rho_{n}$. By Result $3, F / \rho$ is weakly flat and so by assumption is projective. There is, therefore, an $S$-homomorphism $\mu: F / \rho \approx F$ with $v \mu$ the identity map on $F / \rho$, where $\nu$ is the natural $S$-homomorphism $F \rightarrow F / \rho$. Let $\mu\left(x_{1} \rho\right)=c x_{k}(c \in S)$ and for $i>1$ let $\mu\left(x_{i} \rho\right)=\left.c_{i} x_{k}\right|_{i} \mid$. Then

$$
c x_{k}=\mu\left(x_{1} \rho\right)=\mu\left(a_{1} \ldots a_{i-1}\left(x_{i} \rho\right)\right)=a_{1} \ldots a_{i-1} c_{i} x_{k(i)} \text {. }
$$

Hence $k=k(i)$ and $c=a_{1} \ldots a_{i-1} c_{i} \in a_{1} \ldots a_{i-1} S$ so that $c S \subseteq a_{1} \ldots a_{n} S$ for all $n$. But $x_{1} \rho=v \mu\left(x_{1} \rho\right)=\left(c x_{k}\right) \rho$ and $x_{1} \rho a_{1} \ldots a_{k-1} x_{k}$ so that by the first part of the proof $a_{1} \ldots a_{k+m}=c a_{k} \ldots a_{k+m}$ for some $m$. Thus $a_{1} \ldots a_{k+m} S \subseteq c S$ and our descending chain terminates. (v).

The implication (v) $\Rightarrow$ (iii) now follows since by (4), condition $A$ follows from

We next turn our attention to the implications (ii) $\Rightarrow(v)$ and (iii) $\Rightarrow(v)$. Our first objective is to show that in the presence of condition $A$, every indecomposable weakly flat $S$-system is cyclic. Call a generating set $X$ for an $S$-system $A$ independent if for $x, x^{\prime} \in \boldsymbol{X}$ and $x \in S x^{\prime}$ we have $x=x^{\prime}$. For an arbitrary $S$, not every $S$-system has an independent generating set; for example, a non-cyclic, locally cyclic $S$-system does not. However, we have

Lemma 2. Let $A$ be an $S$-system which satisfies the ascending chain condition for cyclic subsystems. If $X$ is a set of generators for $A$, then $X$ contains an independent set of generators for $A$. 
Proof. Call an element $x$ in $X$ basic if whenever $S x \subseteq S x^{\prime}$ with $x^{\prime} \in X$ we have $S x=S x^{\prime}$. Denote by $X^{\prime}$ the set of basic elements in $X$. If $x \in X$, then there is a maximal member $S x^{\prime}$ among the cyclic subsystems of $A$ which contain $S x$. Now $x^{\prime} \in X^{\prime}$ by the maximality of $S x^{\prime}$. Also $x=s x^{\prime}$ for some $s \in S$ and consequently $X^{\prime}$ generates $A$.

If $x, x^{\prime} \in X^{\prime}$ and $x=s x^{\prime}$, then $S x \subseteq S x^{\prime}$ so that $S x=S x^{\prime}$ and $x^{\prime}=t x$ for some $t \in S$. It follows that the relation $\sim$ on $X^{\prime}$ defined by $x \sim x^{\prime}$ if and only if $x=s x^{\prime}$ for some $s \in S$ is an equivalence relation. Now let $X^{\prime \prime}$ be a set of representatives of the $\sim$-classes.

If $x \in X^{\prime}$, then $x \sim x^{\prime}$ for some $x^{\prime} \in X^{\prime \prime}$ so that $x=s x^{\prime}(s \in S)$ and hence $X^{\prime \prime}$ generates $A$. Finally, $X^{\prime \prime}$ is independent.

Lemma 3. Let $A$ be a weakly flat S-system which satisfies the ascending chain condition for cyclic subsystems. If $A$ is indecomposable, then $A$ is cyclic.

Proof. By Lemma 2, $A$ has an independent set of generators $X$. If $x \in X$, let $a \in A$ be such that $S a$ is maximal among those cyclic subsystems of $A$ which contain $S x$. Put $X^{\prime}=\{a\} \cup Y$, where $Y=X \backslash\{y \in X: y \in S a\}$. Clearly $X^{\prime}$ generates $A$ and is independent. If $X^{\prime}$ contains elements other than $a$, then since $A$ is indecomposable we have $\operatorname{San} S X^{\prime \prime} \neq \varnothing$ where $X^{\prime \prime}=X^{\prime} \backslash\{a\}$. Hence $s a=t y$ for some $y \in X^{\prime \prime}, s, t \in S$. But $A$ is weakly flat and so by Result 4, there is an element $z \in A$ and elements $s^{\prime}, t^{\prime} \in S$ such that $a=s^{\prime} z, y=t^{\prime} z$ and $s s^{\prime}=t t^{\prime}$. Thus $S a \subseteq S z$ and $S a=S z$ by the maximality of $S a$, so that $z=s^{\prime \prime} a$ for some $s^{\prime \prime} \in S$. This gives $y=t^{\prime} s^{\prime \prime} a$ contradicting the independence of $X^{\prime}$. Thus $X^{\prime}=\{a\}$ and $A=S a$ is cyclic.

Corollary. If $S$ satisfies condition $A$, then every weakly flat $S$-system is a disjoint union of weakly fat cyclic S-systems.

Proof. Immediate from Lemma 3 and the remark following Result 4.

In view of this corollary and Result 2 , to prove the implications (iii) $\Rightarrow$ (v) and (ii) $\Rightarrow(v)$, it suffices to show that if $S$ satisfies $M_{R}$ or condition $D$, then weakly flat cyclic $S$-systems are projective.

Lemma 4. If $S$ satisfies $M_{R}$, then every weakly flat cyclic $S$-system is projective.

Proof. Let $C$ be a weakly flat cyclic $S$-system. By the Corollary of Result 4, $C \approx S / \rho$, where $\rho$ is a weakly flat left congruence. If $B=1 \rho$, then $B$ is a subsemigroup of $S$ by Result 5 . Let $c \in B$ be such that $c S$ is minimal in the collection of principal right ideals of $S$ generated by elements of $B$. If $a \in B$, then $a \rho c$ so that $a u=c u$ and $u v \in B$ for some elements $u, v \in S$. Hence $d=a u v=c u v \in B$ and $d S \subseteq a S \cap c S$. The minimality of $c S$ gives $c S=d S \subseteq a S$.

Now define $\alpha: S / \rho \rightarrow S c$ by $\alpha(s \rho)=s c$. If $s \rho=t \rho$, then for some elements $u, v$ in $S, s u=t u$ and $u v \in B$. Hence $c S \subseteq u v S$, so that $c=u v w(w \in S)$ and 
$s c=s u v w=t u v w=t c$ giving that $\alpha$ is well-defined. Clearly $\alpha$ is a surjective $S$-homomorphism. Also $\alpha$ is injective, because if $s c=t c$, then

$$
s \rho=s(1 \rho)=s(c \rho)=(s c) \rho=(t c) \rho=t \rho .
$$

Thus $S / \rho \approx S c$. Since $1 \rho c$, it follows that $c$ is idempotent so that, by Result $2, S c$ is projective.

Lemma 5. If $S$ satisfies condition $D$, then every weakly flat cyclic $S$-system is projective.

Proof. As in Lemma 4, a weakly flat cyclic $S$-system is isomorphic to $S / \rho$ for some weakly flat left congruence $\rho$ and $B=1 \rho$ is a left unitary subsemigroup of $S$. Condition $D$ ensures that $B$ has a minimal left ideal $B e$ generated by an idempotent $e$. By (3, Lemma 8.12),eB is a minimal right ideal of $B$. Since $\rho$ is weakly flat, if $a \in B$, then there are elements $u, v \in S$ such that $a u=e u$ and $u v \in B$. Thus $a u v B \subseteq a B \cap e B$ and the minimality of $e B$ gives $e B=a u v B \subseteq a B$. Hence $e S \subseteq a S$ for all $a \in B$ and $S / \rho \approx S e$ as in the proof of Lemma 4.

Finally, to show that the implication (iii) $\Rightarrow$ (iv) holds we have

Lemma 6. If the right S-system A satisfies the descending chain condition for cyclic subsystems, then it also satisfies the descending chain condition for finitely generated subsystems.

Proof. Since $A$ contains minimal cyclic subsystems, the family of subsystems of $A$ which satisfy the descending chain condition for finitely generated subsystems is not empty. This family is partially ordered by inclusion and Zorn's lemma gives a maximal member $K$. If $K \neq A$, then the set of cyclic subsystems of $A$ not contained in $K$ has a minimal member $x S$. Consider the subsystem $K \cup x S$ and let $K_{1} \supseteq K_{2} \supseteq \ldots$ be a descending chain of finitely generated subsystems of $K \cup x S$. If for any $n, K_{n} \subseteq K$, then clearly the chain terminates. We may assume, therefore, that for all $n$ there is an element $y_{n} \in K_{n} \backslash K$. Clearly $y_{n} \in x S$ so that $y_{n} S \subseteq x S$ but $y_{n} S$ is not contained in $K$. The minimality of $x S$ gives $y_{n} S=x S$ and consequently $K_{n} \cap x S=x S$ for all $n$. Given a finite set $X$ of generators for $K_{n}$, we define $K_{n}^{\prime}$ to be the subsystem generated by $X \backslash x S$. Clearly $K_{n}^{\prime} \subseteq K$ and $K_{n}=K_{n}^{\prime} \cup x S$. Further, $K_{n+1}^{\prime} \subseteq K_{n}^{\prime}$ for if $y$ is one of the generators of $K_{n+1}^{\prime}$, then $y \in K_{n+1} \backslash x S$ so that $y \in K_{n} \backslash x S$ and hence $y \in K_{n}^{\prime}$. The chain $K_{1}^{\prime} \supseteq K_{2}^{\prime} \supseteq \ldots$ consists of finitely generated subsystems of $K$ and so terminates. It follows that the chain $K_{1} \supseteq K_{2} \supseteq \ldots$ terminates so that $K \cup x S$ satisfies the descending chain condition for finitely generated subsystems, contradicting the maximality of $K$. Thus $K=A$ and the Lemma is proved.

\section{REFERENCES}

(1) H. BASS, Finitistic dimension and a homological generalization of semi-primary rings, Trans. Amer. Math. Soc. 95 (1960), 466-488. 
(2) J-E. Bıörk, Rings satisfying a minimum condition on principal ideals, $J$. Reine Angew. Math. 236 (1969), 112-119.

(3) A. H. Clifford and G. B. Preston, The algebraic theory of semigroups, Vol. II (Math. Surveys No. 7, Amer. Math. Soc., 1967).

(4) J. R. Isbell, Perfect monoids, Semigroup Forum 2 (1971), 95-118.

(5) M. KIL'P, On homological classification of monoids. Siber. Math.J. 13 (1972), 396-401.

(6) U. KNAUER, Projectivity of acts and Morita equivalence of monoids, Semigroup Forum 3 (1972), 359-370.

(7) B. Stenström, Flatness and localization over monoids, Math. Nachr. 48 (1971), 315-334.

UNIVERSITY OF YORK

HESLINGTON

YoRK Y01 5DD 